\title{
Interaction of Traveling Curved Fronts in Bistable Reaction-Diffusion Equations in $\mathbb{R}^{2}$
}

\author{
Nai-Wei Liu ${ }^{1,2}$ \\ ${ }^{1}$ School of Mathematics, Shandong University, Jinan, Shandong 250100, China \\ ${ }^{2}$ School of Mathematics and Information Science, Yantai University, Yantai, Shandong 264005, China
}

Correspondence should be addressed to Nai-Wei Liu; liunaiwei@aliyun.com

Received 10 February 2017; Accepted 4 April 2017; Published 7 May 2017

Academic Editor: Emir Köksal

Copyright (c) 2017 Nai-Wei Liu. This is an open access article distributed under the Creative Commons Attribution License, which permits unrestricted use, distribution, and reproduction in any medium, provided the original work is properly cited.

We consider the interaction of traveling curved fronts in bistable reaction-diffusion equations in two-dimensional spaces. We first characterize the growth of the traveling curved fronts at infinity; then by constructing appropriate subsolutions and supersolutions, we prove that the solution of the Cauchy problem converges to a pair of diverging traveling curved fronts in $\mathbb{R}^{2}$ under appropriate initial conditions.

\section{Introduction}

In the current paper, we consider the following Cauchy problem:

$$
\begin{aligned}
\frac{\partial u}{\partial t} & =\Delta u+f(u), \quad(x, y) \in \mathbb{R}^{2}, t>0, \\
u(x, y, 0) & =u_{0}(x, y), \quad(x, y) \in \mathbb{R}^{2},
\end{aligned}
$$

where $u_{0} \in C^{1}\left(\mathbb{R}^{2}\right)$ is a bounded initial function and the function $f$ is of bistable type. Concretely, we assume that $f$ satisfies the following:

(F1) $f \in C^{1}(\mathbb{R})$ and $f(0)=f(1)=0, f^{\prime}(0)<0$ and $f^{\prime}(1)<0$,

(F2) $f(s)<0$ and $f^{\prime}(s)<0$ for $s>1 ; f(s)>0$ and $f^{\prime}(s)<$ 0 for $s<0$,

(F3) $\int_{0}^{1} f(s) d s>0$.

Such an example is

$$
f(u)=u(1-u)(u-a), \quad \text { for } 0<a<\frac{1}{2} .
$$

Under assumptions (F1) and (F2), it is clear that there exists a positive constant $\delta(0<\delta<1 / 2)$ such that

$$
f^{\prime}(u) \leq-\gamma_{1}, \quad u \in(-\infty, 2 \delta) \cup(1-2 \delta, \infty),
$$

where $\gamma_{1}:=(1 / 2) \min \left\{-f^{\prime}(0),-f^{\prime}(1)\right\}>0$.

We remark here that the steady states 0 and 1 of (1) are asymptotically stable if (F1)-(F3) hold. In $\mathbb{R}^{1}$, by letting $u(x-$ $\bar{c} t)=\phi(\xi)$ and $\xi=x-\bar{c} t(\bar{c}>0)$, one has

$$
\begin{aligned}
-\phi^{\prime \prime}(\xi)-\bar{c} \phi^{\prime}(\xi)-f(\phi) & =0, \quad \phi^{\prime}(\xi)<0, \quad(\xi \in \mathbb{R}), \\
\lim _{\xi \rightarrow-\infty} \phi(\xi) & =1, \\
\lim _{\xi \rightarrow \infty} \phi(\xi) & =0 .
\end{aligned}
$$

It is well known that (4) has a planar traveling wave front $\phi(\xi)$ which is unique up to phase shift under assumptions (F1)-(F3), with the unique positive traveling wave speed $\bar{c}$. The traveling wave fronts have been widely studied in many fields, such as biology, chemistry, epidemiology, and physics. One can refer to [1-8] for details. Traveling wave fronts are special solutions of (1), which can be used to characterize the invariant set with respect to transition in spaces. 
Without loss of generality, assume that the solution of (1) travels towards $x$-direction and let

$$
u(x, y, t)=V(z, y, t), \quad z=x-c t
$$

then, we can rewrite (1) into

$$
\begin{gathered}
V_{t}-V_{z z}-V_{y y}-c V_{z}-f(V)=0, \\
\quad(z, y) \in \mathbb{R}^{2}, t>0, \\
\left.V\right|_{t=0}=u_{0}, \quad(z, y) \in \mathbb{R}^{2} .
\end{gathered}
$$

For convenience, we denote the solution of Cauchy problem (6) with initial function $V\left(z, y, 0 ; u_{0}\right)=u_{0}(z, y)$ by $V\left(z, y, t ; u_{0}\right)$.

Considering the traveling wave fronts $u(x, y, t)=$ $\Phi(z, y)(z=x-c t)$ of (1) with traveling wave speed $c$ in $x$ direction, then

$$
-\Phi_{z z}-\Phi_{y y}-c \Phi_{z}-f(\Phi)=0, \quad \text { in } \mathbb{R}^{2} .
$$

It is obvious that the solution of (7) is a stationary solution of (6). Let $c \geq \bar{c}$; then $\phi\left((\bar{c} / c)\left(z \pm m_{*} y\right)\right)$ is a solution of (7), where $\phi(\cdot)$ is a solution of (4) and

$$
m_{*}:=\frac{\sqrt{c^{2}-\bar{c}^{2}}}{\bar{c}}>0 .
$$

We call the solution $\Phi(z, y)$ of (7) traveling curved front, since it is nonplanar. By using comparison principle, one has the function

$$
\begin{aligned}
& \varphi(z, y) \\
& :=\max \left\{\phi\left(\frac{\bar{c}}{c}\left(z-m_{*} y\right)\right), \phi\left(\frac{\bar{c}}{c}\left(z+m_{*} y\right)\right)\right\} \\
& \quad=\phi\left(\frac{\bar{c}}{c}\left(z-m_{*}|y|\right)\right)
\end{aligned}
$$

which is a subsolution of (7) with $\varphi_{z}(z, y)<0$ on $\mathbb{R}^{2}$. By using sub- and supersolutions method, Ninomiya and Taniguchi $[9,10]$ proved the existence and global stability of traveling curved fronts for (1).

Theorem 1 (see [9, 10]). Assume that (F1)-(F3) hold. For any $c>\bar{c}$, there exists a traveling curved front $u(x, y, t)=$ $\Phi(z, y)(z=x-c t)$ of (1) such that

$$
\begin{aligned}
& \lim _{\mathscr{R} \rightarrow+\infty} \sup _{z^{2}+y^{2}>\mathscr{R}^{2}}|\Phi(z, y)-\varphi(z, y)|=0, \\
& \Phi(z, y)>\varphi(z, y), \\
& 0<\Phi(z, y)<1, \\
& \Phi_{z}(z, y)<0, \\
& \text { for }(z, y) \in \mathbb{R}^{2} .
\end{aligned}
$$

Furthermore, if $0<\delta_{1}<1 / 2$, there is a constant $\gamma\left(\delta_{1}\right)>0$ such that

$$
\Phi_{z}(z, y) \leq-\gamma\left(\delta_{1}\right), \quad \text { for } \delta_{1} \leq \Phi \leq 1-\delta_{1} .
$$

If $u_{0}(z, y)$ satisfies

$$
\lim _{\mathscr{R} \rightarrow+\infty} \sup _{z^{2}+y^{2}>\mathscr{R}^{2}}\left|u_{0}(z, y)-\Phi(z, y)\right|=0,
$$

then

$$
\lim _{t \rightarrow+\infty} \sup _{(x, y) \in \mathbb{R}^{2}}\left|u\left(x, y, t ; u_{0}\right)-\Phi(x-c t, y)\right|=0,
$$

where $u\left(x, y, t ; u_{0}\right)$ is the solution of the Cauchy problem (1).

It follows from Theorem 1 that (1) has a unique traveling curved front $\Phi(x-c t, y)$ for each $c>\bar{c}$, which is globally stable in the sense of (14). In fact, there are many mathematical models arising in biology, population dynamics, flame propagation, and disease spread which can be described by traveling curved front. For example, Sheng et al. [11] considered the stability of traveling curved fronts (V-shaped) for Allen-Cahn equations, and they in [11] also proved that the traveling curved fronts (V-shaped) are not asymptotically stable under some perturbations. In another paper, by using comparison principle, Sheng [12] studied the existence and stability of time-periodic traveling curved fronts about bistable reaction-diffusion equations in $\mathbb{R}^{3}$. In [13], Wang and $\mathrm{Bu}$ considered traveling curved fronts (nonplanar) for combustion and degenerate Fisher-KPP type reaction-diffusion equations. Ninomiya and Taniguchi [9, $10]$ and Taniguchi $[14,15]$ showed the existence and the stability of traveling curved fronts for Allen-Cahn equations. Furthermore, by constructing some appropriate subsolutions and supersolutions, Hamel et al. [16] considered the existence and the global stability of traveling curved fronts for a model about conical flames. They in [17] established the existence of traveling curved fronts for bistable model by introducing the conical-limiting conditions at infinity. For more interesting results about the existence and stability of traveling curved fronts, one can refer to [18-28].

In addition to the stability results about traveling fronts mentioned above, the interaction between traveling fronts is also an important topic for reaction-diffusion equations. Here, the interaction of traveling fronts means that the solutions of the Cauchy problem converge to a pair of diverging traveling fronts. Recently, there are many results about this problem. Particularly, Fife and McLeod [29, 30] studied the interaction of traveling fronts in one-dimensional space when $t \rightarrow+\infty$. Indeed, they in $[29,30]$ proved that the solutions of the Cauchy problem converge to a single traveling front, a pair of diverging traveling fronts, and a stacked combination of traveling fronts in $\mathbb{R}^{1}$, respectively. Based on comparison principle, Chen [3] developed the squeeze technique to study the interaction and the exponential stability of traveling wave solution for bistable reactiondiffusion equations. Furthermore, Roquejoffre [31] expanded the results in [29] to infinite cylinders. In another paper, Bebernes et al. [32] proved that the solution converges to a pair of diverging traveling fronts in cylindrical domains. We also remark here that there is another form of interaction between traveling fronts, which can be described by the socalled entire solutions. Entire solutions can be used to imply 
the dynamics of two traveling fronts as $t \rightarrow-\infty$; one can refer to [33-37] for related works.

However, the interaction of traveling curved fronts of reaction-diffusion equations in whole spaces $\mathbb{R}^{2}$ is still open. Since two traveling curved fronts traveling towards opposite directions always interact with each other, a natural issue is that whether we can expect that the solution of (1) converges to a pair of diverging traveling curved fronts in $\mathbb{R}^{2}$ under some appropriate initial conditions, which behaves as the interaction of traveling curved fronts. The current paper is devoted to resolving this problem for bistable reactiondiffusion equations in $\mathbb{R}^{2}$.

In this paper, based on comparison principle, we first construct appropriate sub- and supersolutions and then show that the solution of (1) converges to a pair of diverging traveling curved fronts, which will be done in Section 3. Before doing those, by using the asymptotic decay of planar traveling wave fronts, we give some asymptotic estimates for traveling curved fronts at infinity and list the main result in Section 2.

\section{Preliminaries and Main Result}

In this section, we first study the asymptotic behavior of traveling curved front $\Phi(z, y)$ of (1) as $z \rightarrow-\infty$ by using the result of the exponential convergence of one-dimension traveling wave solution $\phi(\xi)$ of (4) at infinity. In fact, it follows from [38] that there exist positive constants $\mathscr{A}$ and $\mathscr{B}$ such that

$$
\begin{array}{r}
\phi(\xi)=\mathscr{A} e^{-\tilde{\mu} \xi}+o\left(e^{-\tilde{\mu} \xi}\right), \quad \text { as } \xi \longrightarrow+\infty, \\
1-\phi(\xi)=\mathscr{B} e^{\tilde{\lambda} \xi}+o\left(e^{\tilde{\lambda} \xi}\right), \quad \text { as } \xi \longrightarrow-\infty,
\end{array}
$$

where $\tilde{\mu}=\left(\sqrt{\bar{c}^{2}-4 f^{\prime}(0)}+\bar{c}\right) / 2$ and $\widetilde{\lambda}=\left(\sqrt{\bar{c}^{2}-4 f^{\prime}(1)}-\bar{c}\right) / 2$. From [34], we see that the planar traveling wave front $\phi$ of (4) satisfies

$$
1-\phi(\xi) \leq k e^{\tilde{\lambda} \xi}, \quad \xi \leq 0
$$

for some $k>0$ and $\tilde{\lambda}$ defined above.

Under conditions (F1)-(F3) and (3), there exists a constant $\gamma_{2}$ with $\gamma_{2}>\gamma_{1}>0$, such that

$$
f(u) \leq \gamma_{2}(1-u)
$$

for $0<1-u \leq \delta$ with $\delta$ as in (3). Furthermore, by virtue of (12), we have

$$
\Phi_{z}(z, y) \leq-\gamma(\delta):=-\gamma_{3}<0
$$

for $\delta \leq \Phi \leq 1-\delta$. Since the traveling wave front $\phi(\xi)$ of (4) possesses invariance up to translation, we assume that traveling wave front $\phi(\xi)$ satisfies

$$
\phi(0)=\theta, \quad \theta \geq 1-\frac{\delta}{2},
$$

and the constant $k$ in (16) satisfies

$$
k \leq \min \left\{\frac{\gamma_{1} \delta}{4\left(\gamma_{2}-\gamma_{1}\right)}, \frac{\delta}{4}\right\} .
$$

We take three positive constants $q_{0}, \mu$, and $M$ satisfying

$$
\begin{aligned}
& \frac{\delta}{4} \leq q_{0} \leq \frac{\delta}{2}, \\
& 0<\mu \leq \min \left\{\frac{\gamma_{1}}{2}, \lambda c\right\}, \\
& M \geq \max \left\{M_{1}, M_{2}\right\},
\end{aligned}
$$

where

$$
\begin{aligned}
& M_{1}:=\frac{k\left(L_{2}+\gamma_{2}\right)+\left(L_{2}+\mu\right) q_{0}}{\gamma_{3} \mu}, \\
& M_{2}:=\frac{\left(L_{3}+\mu\right) q_{0}}{\gamma_{3} \mu}, \\
& L_{2}:=\max _{-\delta \leq u \leq 1-\delta}\left|f^{\prime}(u)\right|, \\
& L_{3}:=\max _{-\delta \leq u \leq 1+\delta}\left|f^{\prime}(u)\right| .
\end{aligned}
$$

By a translation in the $\xi$-direction, we next take

$$
\tilde{\phi}(\xi)=\phi(\xi-M)
$$

where $M$ is defined in (21). Then, we have

$$
\begin{gathered}
1-\tilde{\phi}(\xi+M)=1-\phi(\xi) \leq k e^{\tilde{\lambda} \xi}, \quad \xi \leq 0, \\
\tilde{\phi}(M)=\phi(0)=\theta, \quad \theta \geq 1-\frac{\delta}{2},
\end{gathered}
$$

by view of (16) and (19).

In the following, we consider planar traveling wave front $\widetilde{\phi}(\xi)$ satisfying (24) and (25) instead of the solution $\phi(\xi)$ of (4) and assume that Theorem 1 holds with $\widetilde{\phi}(\xi)$ instead of $\phi(\xi)$ in the definition of $\varphi(z, y)$. For convenience, in the rest of the paper we drop the tilde of $\widetilde{\phi}$ and denote $\widetilde{\phi}(\xi)$ also by $\phi(\xi)$.

By using the asymptotic behavior of planar traveling wave fronts of (4), we immediately obtain the following lemma.

Lemma 2. Assume that $f$ satisfies (F1)-(F3). Then there exist some positive constants $\lambda, k$, and $C$, such that the traveling curved front $\Phi(z, y)$ defined in Theorem 1 satisfies

$$
\begin{aligned}
& 0 \leq 1-\Phi(z+M, y) \leq k e^{\lambda\left(z-m_{*}|y|\right)}, \\
& \quad z \leq 0, y \in \mathbb{R},
\end{aligned}
$$

$\left|\Phi_{z}(z, y)\right| \leq C, \quad(z, y) \in \mathbb{R}^{2}$,

where $M$ is defined in (21) and

$$
\lambda:=\frac{\bar{c}}{c} \tilde{\lambda}=\frac{\bar{c} \sqrt{\bar{c}^{2}-4 f^{\prime}(1)}-\bar{c}^{2}}{2 c}>0 .
$$

Furthermore, there is

$$
\lim _{\mathscr{R} \rightarrow+\infty} \sup _{\left|z-m_{*}\right| y||>\mathscr{R}} \Phi_{z}(z, y)=0 .
$$


Proof. It follows from (9), (11), and (23) that

$$
\begin{aligned}
0 & \leq 1-\Phi(z+M, y) \leq 1-\varphi(z+M, y) \\
& =1-\phi\left(\frac{\bar{c}}{c}\left(z+M-m_{*}|y|\right)\right) \\
& \leq 1-\phi\left(\frac{\bar{c}}{c}\left(z-m_{*}|y|\right)+M\right) .
\end{aligned}
$$

Thus (26) holds for $z \leq 0$ by (24).

Inequality (27) follows from the standard elliptic estimates. Next, we prove that (29) holds. In fact, if (29) is not true, there exist $\varepsilon_{1}>0$ and $\left\{\left(z_{n}, y_{n}\right)\right\}_{n=1}^{\infty}$ satisfying

$$
\begin{array}{r}
\lim _{n \rightarrow \infty}\left|z_{n}+m_{*}\right| y_{n}||=\infty, \\
\Phi_{z}\left(z_{n}, y_{n}\right) \geq \varepsilon_{1} .
\end{array}
$$

Define

$$
\Phi_{n}(z, y)=\Phi\left(z+z_{n}, y+y_{n}\right), \quad \text { in } B_{0}
$$

where

$$
B_{0}:=\left\{(z, y) \in \mathbb{R}^{2} \mid z^{2}+y^{2}<C\right\},
$$

with $C>0$ a given constant. By extracting subsequence of $\Phi_{n}$ and denoting the subsequence also by $\Phi_{n}$, we have

$$
\Phi_{n}(z, y) \longrightarrow \Phi^{*}(z, y), \quad \text { in } C_{\text {loc }}^{2}\left(\mathbb{R}^{2}\right),
$$

where $\Phi^{*}(z, y)$ is a solution of $(7)$. On the other hand, by view of (10) and (11), we have

$$
\begin{aligned}
& \lim _{n \rightarrow \infty} \sup _{\overline{B_{0}}}\left|\Phi_{n}(z, y)-1\right|=0, \\
& \lim _{n \rightarrow \infty} \sup _{\overline{B_{0}}}\left|f\left(\Phi_{n}(z, y)\right)\right|=0 .
\end{aligned}
$$

Thus the strong maximum principle implies

$$
\frac{\partial}{\partial z} \Phi^{*}(z, y)=0, \quad \text { in } B_{0}
$$

Then,

$$
\frac{\partial}{\partial z} \Phi_{n}(z, y) \longrightarrow 0, \quad \text { as } n \longrightarrow \infty
$$

which contradicts the assumption $\Phi_{z}\left(z_{n}, y_{n}\right) \geq \varepsilon_{1}>0$. Thus, we complete the proof.

Our main result is the following.

Theorem 3. For every $c>\bar{c}$, let $\Phi(\cdot, y)$ be the traveling curved front of (1) defined in Theorem 1 with speed $c$. Assume that (F1)-(F3) hold. Then if $u_{0}(x, y) \in(0,1)$ satisfies

$$
\begin{aligned}
u_{0}(x, y) & \geq \Phi(|x|, y), \\
\lim _{\mathscr{R} \rightarrow+\infty} \sup _{x^{2}+y^{2}>\mathscr{R}^{2}}\left|u_{0}(x, y)-\Phi(|x|, y)\right| & =0,
\end{aligned}
$$

there exist positive constants $q, \mu>0$ and $\zeta$, such that, for all $(x, y, t) \in \mathbb{R}^{2} \times[0,+\infty)$, the solution $u\left(x, y, t ; u_{0}\right)$ of (1) satisfies

$$
\begin{aligned}
\Phi(x & -c t+\zeta, y)+\Phi(-x-c t+\zeta, y)-1-q e^{-\mu t} \\
\leq & u\left(x, y, t ; u_{0}\right) \\
\leq & \Phi(x-c t-\zeta, y)+\Phi(-x-c t-\zeta, y)-1 \\
& +q e^{-\mu t} .
\end{aligned}
$$

Furthermore, one has

$$
\lim _{t \rightarrow+\infty}\left|u\left(x, y, t ; u_{0}\right)-1\right|=0
$$

locally uniformly with respect to $(x, y) \in \mathbb{R}^{2}$.

Remark 4. Inequality (39) implies that the $x$-profile of $u\left(x, y, t ; u_{0}\right)$ approaches that of the traveling curved fronts. In particular, it shows that the domain in which $u$ is close to 1 is expanding at the speed of $c$. The phase shift $\zeta$ is a positive constant which will be defined in the proof of Theorem 3 . The similar stability about traveling curved front in cylinder domain is treated in [32].

In the last of this section, we give the definitions of subsolution and supersolutions for (1) in $\mathbb{R}^{2} \times(0,+\infty)$.

Definition 5. If a function $\underline{u}(x, y, t) \in C^{2,1}\left(\mathbb{R}^{2} \times(0,+\infty), \mathbb{R}\right)$ and satisfies

$$
\begin{aligned}
\frac{\partial \underline{u}(x, y, t)}{\partial t} \leq \Delta \underline{u}(x, y, t)+f(\underline{u}(x, y, t)), & \\
(x, y) & \in \mathbb{R}^{2}, t>0,
\end{aligned}
$$

then $\underline{u}(x, y, t)$ is called a subsolution for $(1)$ in $\mathbb{R}^{2} \times(0,+\infty)$. Similarly, by reversing the inequality in (41), we can define a supersolution $\bar{u}(x, y, t)$ for (1).

\section{Proof of Theorem 3}

In this section, we prove the main result by constructing appropriate sub- and supersolutions. In the following lemma, we construct a subsolution for (1).

Lemma 6. Assume that $(F 1)-(F 3)$ hold. Let $c>\bar{c}$. Then the function

$$
\begin{aligned}
\underline{\phi}(x, y, t):= & \Phi\left(x-c t+M\left(1-e^{-\mu t}\right), y\right) \\
& +\Phi\left(-x-c t+M\left(1-e^{-\mu t}\right), y\right)-1 \\
& -q_{0} e^{-\mu t}
\end{aligned}
$$

is a subsolution of (1) on $t \in(0, \infty)$, where $\Phi(\cdot, y)$ is traveling curved front of (1) defined in Theorem 1 and $q_{0}, \mu>0$ are constants defined in (21). 
Proof. Define

$$
\begin{aligned}
\mathscr{F}(\underline{\phi}(x, y, t)):= & \frac{\underline{\phi}(x, y, t)}{\partial t}-\Delta \underline{\phi}(x, y, t) \\
& -f(\underline{\phi}(x, y, t)) .
\end{aligned}
$$

By using the above prepared results, direct calculations give

$$
\begin{aligned}
\mathscr{F} & (\underline{\phi})=\left(M \mu e^{-\mu t}-c\right) \Phi_{z}\left(x-c t+M\left(1-e^{-\mu t}\right), y\right) \\
& +\left(M \mu e^{-\mu t}-c\right) \Phi_{z}\left(-x-c t+M\left(1-e^{-\mu t}\right), y\right) \\
& +q_{0} \mu e^{-\mu t}-\Phi_{z z}\left(x-c t+M\left(1-e^{-\mu t}\right), y\right) \\
& -\Phi_{z z}\left(-x-c t+M\left(1-e^{-\mu t}\right), y\right)-\Phi_{y y}(x-c t \\
& \left.+M\left(1-e^{-\mu t}\right), y\right)-\Phi_{y y}(-x-c t \\
& \left.+M\left(1-e^{-\mu t}\right), y\right) \\
& -f\left(\Phi\left(x-c t+M\left(1-e^{-\mu t}\right), y\right)\right. \\
& \left.+\Phi\left(-x-c t+M\left(1-e^{-\mu t}\right), y\right)-1-q_{0} e^{-\mu t}\right) \\
& =M \mu e^{-\mu t} \Phi_{z}\left(x-c t+M\left(1-e^{-\mu t}\right), y\right) \\
& +M \mu e^{-\mu t} \Phi_{z}\left(-x-c t+M\left(1-e^{-\mu t}\right), y\right) \\
& +q_{0} \mu e^{-\mu t}+f\left(\Phi\left(x-c t+M\left(1-e^{-\mu t}\right), y\right)\right) \\
+ & f\left(\Phi\left(-x-c t+M\left(1-e^{-\mu t}\right), y\right)\right) \\
& +f\left(\Phi\left(x-c t+M\left(1-e^{-\mu t}\right), y\right)\right. \\
& \left.\left.+x-c t+M\left(1-e^{-\mu t}\right), y\right)-1-q_{0} e^{-\mu t}\right) .
\end{aligned}
$$

If $x \geq 0$, we consider two cases $\Phi\left(x-c t+M\left(1-e^{-\mu t}\right), y\right) \in$ $[0, \delta] \cup[1-\delta, 1]$ and $\Phi\left(x-c t+M\left(1-e^{-\mu t}\right), y\right) \in[\delta, 1-\delta]$, respectively.

Case $A\left(\Phi\left(x-c t+M\left(1-e^{-\mu t}\right), y\right) \in[1-\delta, 1]\right)$. By virtue of (3), (21), and (25), we have

$$
\begin{aligned}
f & \left(\Phi\left(x-c t+M\left(1-e^{-\mu t}\right), y\right)\right) \\
& -f\left(\Phi\left(x-c t+M\left(1-e^{-\mu t}\right), y\right)\right. \\
& \left.+\Phi\left(-x-c t+M\left(1-e^{-\mu t}\right), y\right)-1-q_{0} e^{-\mu t}\right) \\
& \leq-\gamma_{1}\left(1-\Phi\left(-x-c t+M\left(1-e^{-\mu t}\right), y\right)\right. \\
& \left.+q_{0} e^{-\mu t}\right),
\end{aligned}
$$

since $0<1-\Phi\left(-x-c t+M\left(1-e^{-\mu t}\right), y\right)+q_{0} e^{-\mu t} \leq 1-$ $\phi(M)+q_{0} \leq \delta$. By using (17) and (25) and the fact that $0<$
$1-\Phi\left(-x-c t+M\left(1-e^{-\mu t}\right), y\right) \leq 1-\phi(M) \leq \delta / 2$ for $x \geq 0$, we have

$$
\begin{aligned}
& f\left(\Phi\left(-x-c t+M\left(1-e^{-\mu t}\right), y\right)\right) \\
& \quad \leq \gamma_{2}\left(1-\Phi\left(-x-c t+M\left(1-e^{-\mu t}\right), y\right)\right) .
\end{aligned}
$$

Thus, we have

$$
\begin{aligned}
& \mathscr{F}(\underline{\phi}) \\
& \leq- \gamma_{1}\left(1-\Phi\left(-x-c t+M\left(1-e^{-\mu t}\right), y\right)+q_{0} e^{-\mu t}\right) \\
&+\gamma_{2}\left(1-\Phi\left(-x-c t+M\left(1-e^{-\mu t}\right), y\right)\right) \\
&+q_{0} \mu e^{-\mu t} \\
& \leq\left(\gamma_{2}-\gamma_{1}\right)\left(1-\Phi\left(-x-c t+M\left(1-e^{-\mu t}\right), y\right)\right) \\
&-\left(\gamma_{1}-\mu\right) q_{0} e^{-\mu t} \\
& \leq k\left(\gamma_{2}-\gamma_{1}\right) e^{\lambda\left(-x-c t-m_{*}|y|\right)}-\left(\gamma_{1}-\mu\right) q_{0} e^{-\mu t} \\
& \leq k\left(\gamma_{2}-\gamma_{1}\right) e^{-\lambda c t}-\left(\gamma_{1}-\mu\right) q_{0} e^{-\mu t} \leq 0 .
\end{aligned}
$$

In the last inequality, we have used the facts (20), (21), and (26).

By a similar argument, we have $F(\underline{\phi}) \leq 0$ for $\Phi(x-c t+$ $\left.M\left(1-e^{-\mu t}\right), y\right) \in[0, \delta]$ with $x \geq 0$.

Case $B\left(\Phi\left(x-c t+M\left(1-e^{-\mu t}\right), y\right) \in[\delta, 1-\delta]\right)$. In a similar way as above, for $x \geq 0$, we have

$$
\begin{aligned}
& f\left(\Phi\left(x-c t+M\left(1-e^{-\mu t}\right), y\right)\right) \\
& \quad-f\left(\Phi\left(x-c t+M\left(1-e^{-\mu t}\right), y\right)\right. \\
& \left.\quad+\Phi\left(-x-c t+M\left(1-e^{-\mu t}\right), y\right)-1-q_{0} e^{-\mu t}\right) \\
& \quad \leq L_{2}\left(1-\Phi\left(-x-c t+M\left(1-e^{-\mu t}\right), y\right)\right. \\
& \left.\quad+q_{0} e^{-\mu t}\right)
\end{aligned}
$$

where $L_{2}:=\max _{-\delta \leq u \leq 1-\delta}\left|f^{\prime}(u)\right|$. Particularly, we have (46) in this case. Lastly, due to (18), we have

$$
\begin{aligned}
& \Phi_{z}^{\prime}\left(x-c t+M\left(1-e^{-\mu t}\right), y\right) \\
& \quad+\Phi_{z}^{\prime}\left(-x-c t+M\left(1-e^{-\mu t}\right), y\right) \leq-\gamma_{3} .
\end{aligned}
$$

Combining (46), (48), and (49), we have

$$
\begin{aligned}
\mathscr{F}(\underline{\phi}) \leq-\gamma_{3} M \mu e^{-\mu t}+L_{2}(1 \\
\left.\quad-\Phi\left(-x-c t+M\left(1-e^{-\mu t}\right), y\right)+q_{0} e^{-\mu t}\right)+\gamma_{2}(1 \\
\left.\quad-\Phi\left(-x-c t+M\left(1-e^{-\mu t}\right), y\right)\right)+q_{0} \mu e^{-\mu t}
\end{aligned}
$$




$$
\begin{aligned}
& \leq-\gamma_{3} M \mu e^{-\mu t}+\left(L_{2}+\gamma_{2}\right)(1 \\
& \left.-\Phi\left(-x-c t+M\left(1-e^{-\mu t}\right), y\right)\right)+\left(L_{2}+\mu\right) \\
& \cdot q_{0} e^{-\mu t} \leq-\gamma_{3} M \mu e^{-\mu t}+k\left(L_{2}+\gamma_{2}\right) e^{-\lambda c t}+\left(L_{2}\right. \\
& +\mu) q_{0} e^{-\mu t}
\end{aligned}
$$

Consequently, (21) implies $\mathscr{F}(\phi) \leq 0$ for this case.

Similarly, we can prove $\overline{\mathscr{F}}(\phi) \leq 0$ when $x<0$. Thus, we have showed that $\phi(x, y, t)$ is a subsolution of (1) on $t \epsilon$ $[0, \infty)$.

In order to construct a supersolution for (1), we introduce the following lemmas.

Lemma 7. Assume that (F1)-(F3) hold. Let $c>\bar{c}$; then for any given constant $N>0$, the function

$$
\begin{aligned}
\phi_{N}^{1}(x, y, t):= & \Phi\left(x-c t-M\left(1-e^{-\mu t}\right)-N, y\right) \\
& +q_{0} e^{-\mu t}
\end{aligned}
$$

is a supersolution of (1) on $t \in(0,+\infty)$, where $\Phi(\cdot, y)$ is traveling curved front of (1) as in Theorem 1 and $q_{0}, \mu>0$ are constants defined in (21).

Proof. As the proof of Lemma 6, we need only to prove that the right hand of (43) is nonnegative for the function $\phi_{N}^{1}(x, y, t)$ for $(x, y, t) \in \mathbb{R}^{2} \times(0,+\infty)$. By a similar argument, direct calculations give

$$
\begin{aligned}
\mathscr{F} & \left(\phi_{N}^{1}\right) \\
= & \left(-M \mu e^{-\mu t}-c\right) \Phi_{z}\left(x-c t-M\left(1-e^{-\mu t}\right)-N, y\right) \\
& -q_{0} \mu e^{-\mu t}-\Phi_{z z}\left(x-c t-M\left(1-e^{-\mu t}\right)-N, y\right) \\
& -\Phi_{y y}\left(x-c t-M\left(1-e^{-\mu t}\right)-N, y\right) \\
& -f\left(\Phi\left(x-c t-M\left(1-e^{-\mu t}\right)-N, y\right)+q_{0} e^{-\mu t}\right) \\
= & -M \mu e^{-\mu t} \Phi \Phi_{z}\left(x-c t-M\left(1-e^{-\mu t}\right)-N, y\right) \\
& -q_{0} \mu e^{-\mu t} \\
& +f\left(\Phi\left(x-c t-M\left(1-e^{-\mu t}\right)-N, y\right)\right) \\
& -f\left(\Phi\left(x-c t-M\left(1-e^{-\mu t}\right)-N, y\right)+q_{0} e^{-\mu t}\right) .
\end{aligned}
$$

To complete the proof, we consider two cases $\Phi(x-c t-$ $\left.M\left(1-e^{-\mu t}\right)-N, y\right) \in[0, \delta] \cup[1-\delta, 1]$ and $\Phi(x-c t-M(1-$ $\left.\left.e^{-\mu t}\right)-N, y\right) \in[\delta, 1-\delta]$, respectively.

For $\Phi\left(x-c t-M\left(1-e^{-\mu t}\right)-N, y\right) \in[0, \delta] \cup[1-\delta, 1]$, we just consider the case $\Phi\left(x-c t-M\left(1-e^{-\mu t}\right)-N, y\right) \in[1-\delta, 1]$.
Since $\Phi\left(x-c t-M\left(1-e^{-\mu t}\right)-N, y\right)+q_{0} e^{-\mu t} \in[1-\delta, 1+\delta]$, it follows from (3) that

$$
\begin{aligned}
f( & \left.\left(x-c t-M\left(1-e^{-\mu t}\right)-N, y\right)+q_{0} e^{-\mu t}\right) \\
& -f\left(\Phi\left(x-c t-M\left(1-e^{-\mu t}\right)-N, y\right)\right) \\
\leq & -\gamma_{1} q_{0} e^{-\mu t} .
\end{aligned}
$$

By virtue of (11) and (21), we have

$$
\begin{aligned}
\mathscr{F}\left(\phi_{N}^{1}\right) & \\
\geq & -M \mu e^{-\mu t} \Phi_{z}\left(x-c t-M\left(1-e^{-\mu t}\right)-N, y\right) \\
& \quad-q_{0} \mu e^{-\mu t}+\gamma_{1} q_{0} e^{-\mu t} \geq q_{0}\left(\gamma_{1}-\mu\right) e^{-\mu t} \geq 0 .
\end{aligned}
$$

For $\Phi\left(x-c t-M\left(1-e^{-\mu t}\right)-N, y\right) \in[\delta, 1-\delta]$, we have

$$
\begin{aligned}
f( & \left.\left(x-c t-M\left(1-e^{-\mu t}\right)-N, y\right)\right) \\
& -f\left(\Phi\left(x-c t-M\left(1-e^{-\mu t}\right)-N, y\right)+q_{0} e^{-\mu t}\right) \\
\geq & -L_{3} q_{0} e^{-\mu t},
\end{aligned}
$$

where $L_{3}:=\max _{-\delta \leq u \leq 1+\delta}\left|f^{\prime}(u)\right|$. Particularly, (18) implies

$$
\Phi_{z}^{\prime}\left(x-c t-M\left(1-e^{-\mu t}\right)-N, y\right) \leq-\gamma_{3} .
$$

Therefore, by (21) we get

$$
\begin{aligned}
\mathscr{F}\left(\phi_{N}^{1}\right) & \geq \gamma_{3} M \mu e^{-\mu t}-q_{0} \mu e^{-\mu t}-L_{3} q_{0} e^{-\mu t} \\
& \geq \gamma_{3} M \mu e^{-\mu t}-\left(L_{3}+\mu\right) q_{0} e^{-\mu t} \geq 0 .
\end{aligned}
$$

Thus, $\phi_{N}^{1}(x, y, t)$ defined by (51) is a supersolution of (1).

In a similar way, we prove the following lemma.

Lemma 8. Assume that (F1)-(F3) hold. Let $c>\bar{c}$; then for any given constant $N>0$, the function

$$
\begin{aligned}
\phi_{N}^{2}(x, y, t):= & \Phi\left(-x-c t-M\left(1-e^{-\mu t}\right)-N, y\right) \\
& +q_{0} e^{-\mu t}
\end{aligned}
$$

is a supersolution of (1) on $t \in(0,+\infty)$, where $\Phi(\cdot, y)$ is traveling curved front of (1) as in Theorem 1 and $q_{0}, \mu>0$ are constants defined in (21).

Remark 9. Let $c>\bar{c}$ and $N>0$; it follows from Lemmas 7 and 8 that the function

$$
\begin{aligned}
\bar{\phi}_{N}(x, y, t):= & \Phi\left(|x|-c t-M\left(1-e^{-\mu t}\right)-N, y\right) \\
& +q_{0} e^{-\mu t}
\end{aligned}
$$

is a supersolution of $(1)$ on $t \in(0,+\infty)$.

To complete the proof of Theorem 3, we establish the following comparison result. 
Lemma 10. Let $\phi(x, y, t)$ and $\bar{\phi}_{N}(x, y, t)$ be defined by (42) and (59), respectively, and $u_{0}(x, y) \in(0,1)$ satisfies (38). Then, there exists $N>0$ such that

$$
\underline{\phi}(x, y, t) \leq u\left(x, y, t ; u_{0}\right) \leq \bar{\phi}_{N}(x, y, t)
$$

for all $(x, y, t) \in \mathbb{R}^{2} \times[0,+\infty)$.

Proof. By (38) and the definition of (42), when $t=0$, direct calculations give

$$
\begin{aligned}
\underline{\phi}(x, y, 0) & =\Phi(x, y)+\Phi(-x, y)-1-q_{0} \leq \Phi(x, y) \\
& \leq u_{0}(x, y)
\end{aligned}
$$

for $x \geq 0$. Similarly, we have $\phi(x, y, 0) \leq u_{0}(x, y)$ for $x<$ 0 . Therefore, the maximum principle for parabolic equations shows $\phi(x, y, t) \leq u\left(x, y, t ; u_{0}\right)$ for $(x, y, t) \in \mathbb{R}^{2} \times[0,+\infty)$.

Similarly, by the definition of (59), when $t=0$

$$
\bar{\phi}_{N}(x, y, 0)=\Phi(|x|-N, y)+q_{0} .
$$

By (38), we have that, for $\varepsilon=q_{0}$ and $N_{1} \geq 0$, there exists $\Lambda>0$ such that

$$
\begin{aligned}
u_{0}(x, y) & \leq \Phi(|x|, y)+q_{0} \leq \Phi\left(|x|-N_{1}, y\right)+q_{0} \\
& \leq \bar{\phi}_{N_{1}}(x, y, 0)
\end{aligned}
$$

in $\left\{(x, y) \mid x^{2}+y^{2}>\Lambda^{2}\right\}$, since $\Phi_{z}(z, y)<0$.

In the range of $\left\{(x, y) \mid x^{2}+y^{2} \leq \Lambda^{2}\right\}$, we have $|x| \leq \Lambda$. Thus, by choosing $N_{2} \geq \Lambda-M$ and using (20), (21), and (26), we have

$$
\begin{aligned}
\bar{\phi}_{N_{2}}(x, y, 0) & \geq 1-k e^{\lambda\left(|x|-M-N_{2}-m_{*}|y|\right)}+q_{0} \\
& \geq 1-k e^{\lambda\left(\Lambda-M-(\Lambda-M)-m_{*}|y|\right)}+q_{0} \\
& \geq 1-k+q_{0} \geq 1
\end{aligned}
$$

for $(x, y) \in\left\{(x, y) \mid x^{2}+y^{2} \leq \Lambda^{2}\right\}$. Consequently, we have $\bar{\phi}_{N_{2}}(x, y, 0) \geq u_{0}(x, y) \in(0,1)$ in this range.

Combining (63) and (64) and taking $N \geq \max \left\{N_{1}, N_{2}\right\}$, we conclude that $u_{0}(x, y) \leq \bar{\phi}_{N}(x, y, 0)$ for $(x, y) \in \mathbb{R}^{2}$. Then the maximum principle for parabolic equations derives that (60) holds.

Proof of Theorem 3. It follows from Lemma 10 that (60) holds with $N \geq \max \left\{N_{1}, N_{2}\right\}$. Thus, by (11), we obtain

$$
\begin{aligned}
\underline{\phi}(x, y, t)= & \Phi\left(x-c t+M\left(1-e^{-\mu t}\right), y\right) \\
& +\Phi\left(-x-c t+M\left(1-e^{-\mu t}\right), y\right)-1 \\
& -q_{0} e^{-\mu t} \\
\geq & \Phi\left(x-c t+\zeta_{1}, y\right)+\Phi\left(-x-c t+\zeta_{1}, y\right) \\
& -1-q_{0} e^{-\mu t}
\end{aligned}
$$

for all $(x, y, t) \in \mathbb{R}^{2} \times[0,+\infty)$, if $\zeta_{1} \geq M$. On the other hand, for $x \geq 0$, we have

$$
\begin{aligned}
& \bar{\phi}_{N}(x, y, t)=\Phi\left(x-c t-M\left(1-e^{-\mu t}\right)-N, y\right) \\
& +q_{0} e^{-\mu t} \\
& \leq \Phi\left(x-c t-M\left(1-e^{-\mu t}\right)-N, y\right) \\
& +\Phi\left(-x-c t-M\left(1-e^{-\mu t}\right)-N, y\right) \\
& -1+q_{0} e^{-\mu t}+1 \\
& -\Phi\left(-x-c t-M\left(1-e^{-\mu t}\right)-N, y\right) \\
& \leq \Phi\left(x-c t-M\left(1-e^{-\mu t}\right)-N, y\right) \\
& +\Phi\left(-x-c t-M\left(1-e^{-\mu t}\right)-N, y\right) \\
& -1+q_{0} e^{-\mu t}+k e^{\lambda\left(-x-c t-M-N-m_{*}|y|\right)} \\
& \leq \Phi\left(x-c t-M\left(1-e^{-\mu t}\right)-N, y\right) \\
& +\Phi\left(-x-c t-M\left(1-e^{-\mu t}\right)-N, y\right) \\
& -1+q_{0} e^{-\mu t}+k e^{-\lambda c t} \\
& \leq \Phi\left(x-c t-M\left(1-e^{-\mu t}\right)-N, y\right) \\
& +\Phi\left(-x-c t-M\left(1-e^{-\mu t}\right)-N, y\right) \\
& -1+\left(q_{0}+k\right) e^{-\mu t} \\
& \leq \Phi\left(x-c t-\zeta_{2}, y\right)+\Phi\left(-x-c t-\zeta_{2}, y\right) \\
& -1+q e^{-\mu t}
\end{aligned}
$$

if $\zeta_{2} \geq M+N$ and $q \geq\left(q_{0}+k\right)$. By a similar argument, for $x<0,(66)$ also holds.

Thus, if $u_{0}(x, y) \in(0,1)$ satisfies $(38)$, by taking $\zeta=$ $\max \left\{\zeta_{1}, \zeta_{1}\right\}$ and $q \geq\left(q_{0}+k\right)$, we obtain that (39) holds, where $q_{0}$ and $\mu>0$ are defined in (21).

The asymptotic behavior (40) immediately follows from (39). This completes the proof of Theorem 3.

\section{Discussion}

In the current paper, we have proved that the solutions of the bistable reaction-diffusion equations converge to a pair of diverging traveling curved fronts in $\mathbb{R}^{2}$. It means that the solution $u\left(x, y, t, u_{0}\right)$ of (1) with initial function $u_{0}(x, y)$ satisfied (38) behaving as two traveling curved fronts traveling towards opposite directions and approaching each other. Our result is different from the stability results in [9-11, 22, 26, 27]. Indeed, the interaction between traveling wave fronts plays an important role in the study of reaction-diffusion equations in $\mathbb{R}^{2}$, which is crucially related to the pattern formation problem, and there are important applications in chemical, physical, biological systems; see, for example, [39-41]. 
At last, we note here that the global exponential stability of traveling curved fronts in the sense of Theorem 3 is a difficult problem, since the level set of the traveling curved fronts $\Phi(z, y)$ of (1) have two asymptotic directions as $|z| \rightarrow+\infty$, and both directions make an angle with the negative $y$-axis, which is different from the case of planar traveling fronts (see [20]). We will leave it for a further study. Moreover, how the solution of (1) approaches a "stacked" combination of traveling curved fronts just as the study in Fife and McLeod [29] is also an interesting problem but remains open.

\section{Conflicts of Interest}

The author declares that they have no conflicts of interest.

\section{Acknowledgments}

The author is very grateful to Dr. Wei-Jie Sheng for helpful discussions. The author's work was partially supported by NSF of China (11371179 and 11401513) and by China Postdoctoral Science Foundation Funded Project (2014M560546).

\section{References}

[1] D. G. Aronson and H. F. Weinberger, "Multidimensional nonlinear diffusion arising in population genetics," Advances in Mathematics, vol. 30, no. 1, pp. 33-76, 1978.

[2] N. F. Britton, Reaction-Diffusion Equations and Their Applications to Biology, Academic Press, San Diego, SC, USA, 1986.

[3] X. Chen, "Existence, uniqueness, and asymptotic stability of traveling waves in nonlocal evolution equations," Advances in Differential Equations, vol. 2, no. 1, pp. 125-160, 1997.

[4] P. C. Fife, Mathematical Aspects of Reacting and Diffusing Systems, vol. 28 of Lecture Notes in Biomathematics, Springer, Berlin, Germany, 1979.

[5] J. D. Murray, Mathematical Biology, Springer, Berlin, Germany, 1989.

[6] P. Pelcé, Dynamics of Curved Fronts, Academic Press Inc., San Diego, SC, USA, 1988.

[7] A. I. Volpert, V. A. Volpert, and V. A. Volpert, Traveling Wave Solutions of Parabolic Systems, vol. 140 of Translations of Mathematical Monographs, American Mathematical Society, Rhode Island, RI, USA, 1994.

[8] J. Wu, Theory and Applications of Partial Functional-Differential Equations, Springer, New York, NY, USA, 1996.

[9] H. Ninomiya and M. Taniguchi, "Existence and global stability of traveling curved fronts in the Allen-Cahn equations," Journal of Differential Equations, vol. 213, no. 1, pp. 204-233, 2005.

[10] H. Ninomiya and M. Taniguchi, "Global stability of traveling curved fronts in the Allen-Cahn equations," Discrete and Continuous Dynamical Systems. Series A, vol. 15, no. 3, pp. 819832, 2006.

[11] W. Sheng, W. Li, and Z. Wang, "Multidimensional stability of V-shaped traveling fronts in the Allen-Cahn equation," Science China. Mathematics, vol. 56, no. 10, pp. 1969-1982, 2013.

[12] W.-J. Sheng, “Time periodic traveling curved fronts of bistable reaction-diffusion equations in $\mathbb{R}^{3}$," Annali di Matematica Pura ed Applicata. Series IV, vol. 196, no. 2, pp. 617-639, 2017.

[13] Z.-C. Wang and Z.-H. Bu, "Nonplanar traveling fronts in reaction-diffusion equations with combustion and degenerate
Fisher-KPP nonlinearities," Journal of Differential Equations, vol. 260, no. 7, pp. 6405-6450, 2016.

[14] M. Taniguchi, "Traveling fronts of pyramidal shapes in the Allen-Cahn equations," SIAM Journal on Mathematical Analysis, vol. 39, no. 1, pp. 319-344, 2007.

[15] M. Taniguchi, "The uniqueness and asymptotic stability of pyramidal traveling fronts in the Allen-Cahn equations," Journal of Differential Equations, vol. 246, no. 5, pp. 2103-2130, 2009.

[16] F. Hamel, R. Monneau, and J.-M. Roquejoffre, "Stability of travelling waves in a model for conical flames in two space dimensions," Annales Scientifiques de l'École Normale Supérieure. Quatrième Série, vol. 37, no. 3, pp. 469-506, 2004.

[17] F. Hamel, R. Monneau, and J.-M. Roquejoffre, "Existence and qualitative properties of multidimensional conical bistable fronts," Discrete and Continuous Dynamical Systems. Series A, vol. 13, no. 4, pp. 1069-1096, 2005.

[18] X. Chen, J.-S. Guo, F. Hamel, H. Ninomiya, and J.-M. Roquejoffre, "Traveling waves with paraboloid like interfaces for balanced bistable dynamics," Annales de l'Institut Henri Poincaré. Analyse Non Linéaire, vol. 24, no. 3, pp. 369-393, 2007.

[19] C. Gui, "Symmetry of traveling wave solutions to the AllenCahn equation in $\mathbb{R}^{2}$," Archive for Rational Mechanics and Analysis, vol. 203, no. 3, pp. 1037-1065, 2012.

[20] F. Hamel, R. Monneau, and J.-M. Roquejoffre, "Asymptotic properties and classification of bistable fronts with Lipschitz level sets," Discrete and Continuous Dynamical Systems, vol. 14, no. 1, pp. 75-92, 2006.

[21] Y. Kurokawa and M. Taniguchi, "Multi-dimensional pyramidal travelling fronts in the Allen-Cahn equations," Proceedings of the Royal Society of Edinburgh. Section A. Mathematics, vol. 141, no. 5, pp. 1031-1054, 2011.

[22] W.-J. Sheng, W.-T. Li, and Z.-C. Wang, "Periodic pyramidal traveling fronts of bistable reaction-diffusion equations with time-periodic nonlinearity," Journal of Differential Equations, vol. 252, no. 3, pp. 2388-2424, 2012.

[23] W.-J. Sheng, "Time periodic traveling curved fronts of bistable reaction-diffusion equations in $\mathbb{R}^{N}$," Applied Mathematics Letters. An International Journal of Rapid Publication, vol. 54, pp. 22-30, 2016.

[24] M. Taniguchi, "Multi-dimensional traveling fronts in bistable reaction-diffusion equations," Discrete and Continuous Dynamical Systems. Series A, vol. 32, no. 3, pp. 1011-1046, 2012.

[25] M. Taniguchi, "An $(N-1)$-dimensional convex compact set gives an $N$-dimensional traveling front in the Allen-Cahn equation," SIAM Journal on Mathematical Analysis, vol. 47, no. 1, pp. 455-476, 2015.

[26] Z.-C. Wang and J. Wu, "Periodic traveling curved fronts in reaction-diffusion equation with bistable time-periodic nonlinearity," Journal of Differential Equations, vol. 250, no. 7, pp. 31963229, 2011.

[27] Z.-C. Wang, "Traveling curved fronts in monotone bistable systems," Discrete and Continuous Dynamical Systems. Series A, vol. 32, no. 6, pp. 2339-2374, 2012.

[28] Z.-C. Wang, "Cylindrically symmetric travelling fronts in a periodic reaction-diffusion equation with bistable nonlinearity," Proceedings of the Royal Society of Edinburgh. Section A. Mathematics, vol. 145, no. 5, pp. 1053-1090, 2015.

[29] P. C. Fife and J. B. McLeod, "The approach of solutions of nonlinear diffusion equations to travelling front solutions," Archive for Rational Mechanics and Analysis, vol. 65, no. 4, pp. 335-361, 1977. 
[30] P. C. Fife and J. B. McLeod, "A phase plane discussion of convergence to travelling fronts for nonlinear diffusion," Archive for Rational Mechanics and Analysis, vol. 75, no. 4, pp. 281-314, 1981.

[31] J.-M. Roquejoffre, "Eventual monotonicity and convergence to travelling fronts for the solutions of parabolic equations in cylinders," Annales de l'Institut Henri Poincaré. Analyse Non Linéaire, vol. 14, no. 4, pp. 499-552, 1997.

[32] J. W. Bebernes, C. Li, and Y. Li, "Travelling fronts in cylinders and their stability," The Rocky Mountain Journal of Mathematics, vol. 27, no. 1, pp. 123-150, 1997.

[33] X. Chen and J.-S. Guo, "Existence and uniqueness of entire solutions for a reaction-diffusion equation," Journal of Differential Equations, vol. 212, no. 1, pp. 62-84, 2005.

[34] J.-S. Guo and Y. Morita, "Entire solutions of reaction-diffusion equations and an application to discrete diffusive equations," Discrete and Continuous Dynamical Systems, vol. 12, no. 2, pp. 193-212, 2005.

[35] F. Hamel and N. Nadirashvili, "Entire solutions of the KPP equation," Communications on Pure and Applied Mathematics, vol. 52, no. 10, pp. 1255-1276, 1999.

[36] W.-T. Li, N.-W. Liu, and Z.-C. Wang, "Entire solutions in reaction-advection-diffusion equations in cylinders," Journal de Mathématiques Pures et Appliquées. Neuvième Série, vol. 90, no. 5, pp. 492-504, 2008.

[37] N.-W. Liu, W.-T. Li, and Z.-C. Wang, "Entire solutions of reaction-advection-diffusion equations with bistable nonlinearity in cylinders," Journal of Differential Equations, vol. 246, no. 11, pp. 4249-4267, 2009.

[38] H. Berestycki and L. Nirenberg, "Travelling fronts in cylinders," Annales de l'Institut Henri Poincaré. Analyse Non Linéaire, vol. 9, no. 5, pp. 497-572, 1992.

[39] S.-I. Ei, "The motion of weakly interacting pulses in reactiondiffusion systems," Journal of Dynamics and Differential Equations, vol. 14, no. 1, pp. 85-137, 2002.

[40] S.-I. Ei, M. Mimura, and M. Nagayama, "Pulse-pulse interaction in reaction-diffusion systems," Physica D. Nonlinear Phenomena, vol. 165, no. 3-4, pp. 176-198, 2002.

[41] T. Kawahara and M. Tanaka, "Interactions of traveling fronts: an exact solution of a nonlinear diffusion equation," Physics Letters. A, vol. 97, no. 8, pp. 311-314, 1983. 


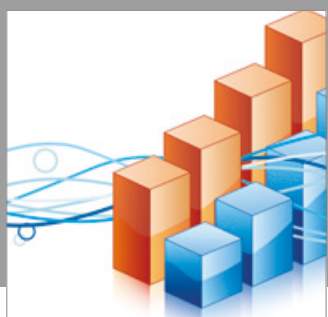

Advances in

Operations Research

vatersals

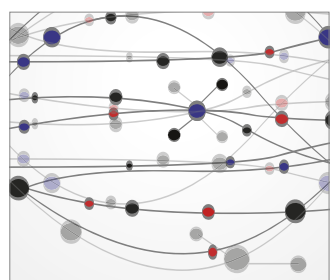

\section{The Scientific} World Journal
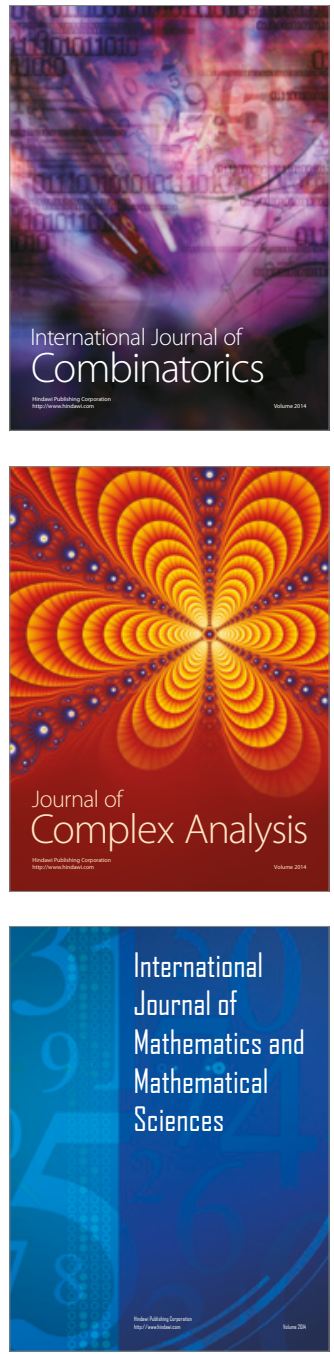
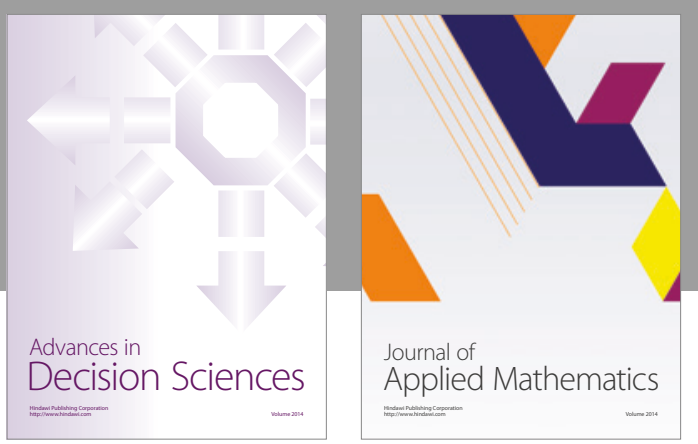

Algebra

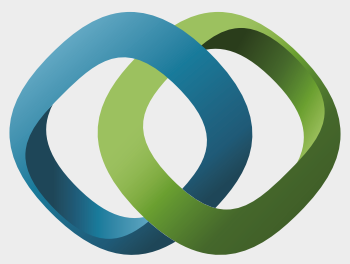

\section{Hindawi}

Submit your manuscripts at

https://www.hindawi.com
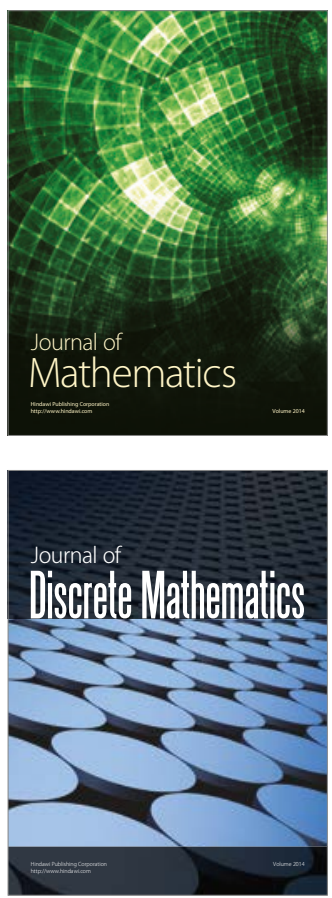

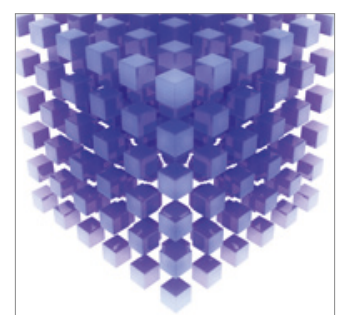

Mathematical Problems in Engineering
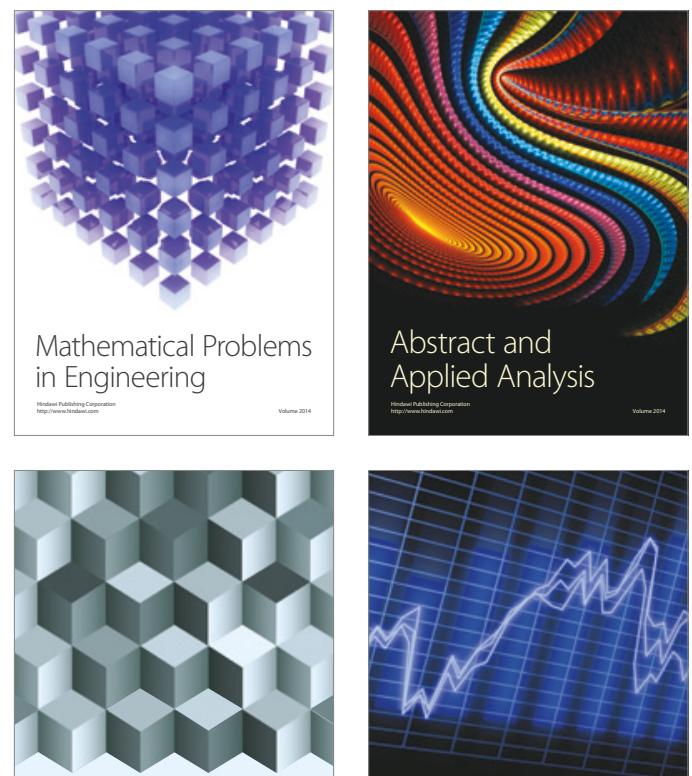

Journal of

Function Spaces

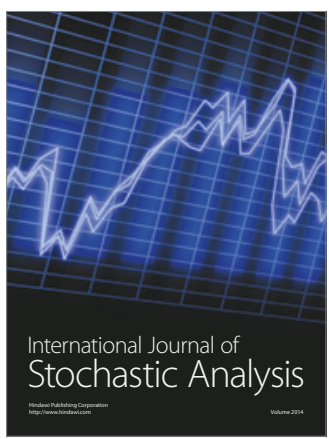

Probability and Statistics
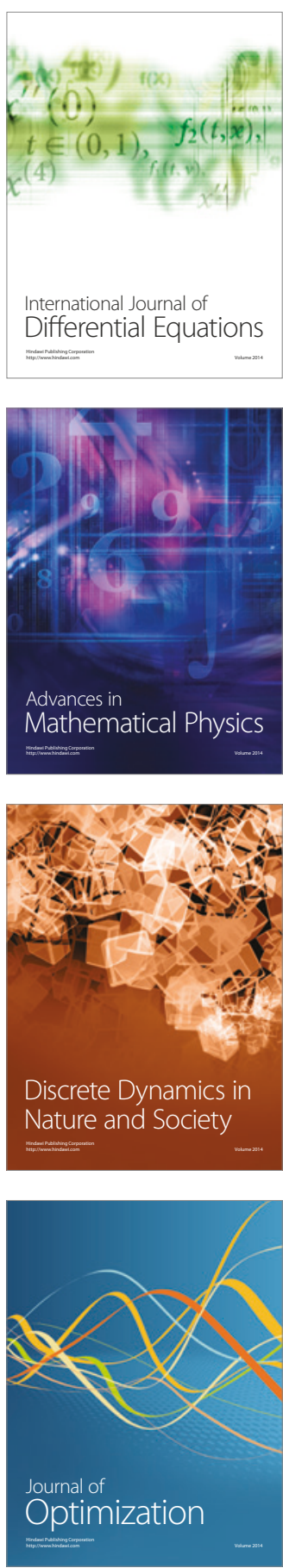